April 2002

\title{
The analytical values of the sunrise master integrals for one of the masses equal to zero.
}

\author{
H. Czyż , A. Grzelińska and R. Zabawa \\ Institute of Physics, University of Silesia, PL-40007 Katowice, Poland \\ e-mail: czyz@us.edu.pl \\ grzel@joy.phys.us.edu.pl
}

\begin{abstract}
The analytical values of the sunrise master amplitudes were found for one mass equal to zero, two other arbitrary masses and an arbitrary external four momentum. Differential equations in the square of the external four momentum and masses were used to obtain the master integrals.
\end{abstract}

keywords: sunrise diagram, analytical calculations, master integrals.

PACS 11.10.-z Field theory

PACS 11.10.Kk Field theories in dimensions other than four

PACS 11.15.Bt General properties of perturbation theory 


\section{Introduction.}

High precision measurements in elementary particle physics require more and more precise calculations of multi-loop Feynman diagrams allowing in the same time detailed tests of the existing models.

The widely used integration by part technique [1] allows for reduction of the complicated task of calculation of radiative corrections to calculation of a limited number of scalar integrals called master integrals (MI). Whenever possible it is of great importance to know analytically that basic building blocks, as it safes enormously the CPU time required for their numerical evaluation. Investigations of the sunrise two-loop diagram started a long time ago and it is beyond the scope of that paper to present the complete literature of the subject, so we will give here only a partial guideline of the literature sending the reader to the references of the quoted paper for further studies.

The MI of the sunrise graph were found to be expressible as a combination of Lauricella functions in [2]. Their analytical expansions at $p^{2}=0$ and $p^{2}=\infty$ were given in [2] and [3]. Their values at threshold $p^{2}=-\left(m_{1}+m_{2}+m_{3}\right)^{2}$ and pseudothresholds $\left(p^{2}=\right.$ $\left.-\left(m_{1}-m_{2}+m_{3}\right)^{2}, p^{2}=-\left(m_{1}+m_{2}-m_{3}\right)^{2}, p^{2}=-\left(-m_{1}+m_{2}+m_{3}\right)^{2}\right)$ were presented in [四]. The analytical expansions at pseudothresholds were found in [5]. A semi-analytical expansion at threshold was given in [6] and also using the configuration space technique in [7], while the complete analytical expansion at threshold was presented in [8]. Some special values of the sunrise MI were obtained in [9] and [10].

\section{Calculation of the sunrise master integrals.}

The scalar integrals related to the sunrise two-loop graph can be defined as [3]

$$
\begin{aligned}
& A\left(n, m_{1}^{2}, m_{2}^{2}, m_{3}^{2}, p^{2},-\alpha_{1},-\alpha_{2},-\alpha_{3}, \beta_{1}, \beta_{2}\right)=\frac{1}{\left((2 \pi)^{n-2}\right)^{2}} \\
& \quad \int d^{n} k_{1} \int d^{n} k_{2} \frac{\left(p \cdot k_{1}\right)^{\beta_{1}}\left(p \cdot k_{2}\right)^{\beta_{2}}}{\left(k_{1}^{2}+m_{1}^{2}\right)^{\alpha_{1}}\left(k_{2}^{2}+m_{2}^{2}\right)^{\alpha_{2}}\left(\left(p-k_{1}-k_{2}\right)^{2}+m_{3}^{2}\right)^{\alpha_{3}}}
\end{aligned}
$$

where $m_{i}(i=1,2,3)$ are the masses associated with internal lines, $p$ is the external momentum, $k_{1}, k_{2}$ are loop momenta and $\alpha_{i}, \quad(i=1,2,3), \beta_{j}, \quad(j=1,2)$ are integer numbers. The integrals are to be performed in $n$-dimensional Euclidean space. It means 
we use the dimensional regularization and have performed the Wick rotation. The scale parameter $\mu$ associated with the dimensional regularization was set to 1 . Final results can be easily rewritten in Minkowski space by changing $p^{2} \rightarrow-p^{2}$.

The four independent Master Integrals (MI) were chosen as

$$
\begin{aligned}
& F_{j}\left(n, m_{1}^{2}, m_{2}^{2}, m_{3}^{2}, p^{2}\right)=\frac{1}{\left((2 \pi)^{n-2}\right)^{2}} \\
& \quad \int d^{n} k_{1} \int d^{n} k_{2} \frac{1}{\left(k_{1}^{2}+m_{1}^{2}\right)^{\alpha_{1}(j)}\left(k_{2}^{2}+m_{2}^{2}\right)^{\alpha_{2}(j)}\left(\left(p-k_{1}-k_{2}\right)^{2}+m_{3}^{2}\right)^{\alpha_{3}(j)}},
\end{aligned}
$$

with $j=0,1,2,3 ; i=1,2,3 ; \alpha_{i}(0)=1 ; \alpha_{i}(j)=1$, for $j \neq i ; \alpha_{i}(j)=2$, for $j=i$. While their $(n-4)$ expansions are defined [3] by

$$
\begin{aligned}
F_{j}\left(n, m_{1}^{2}, m_{2}^{2}, m_{3}^{2}, p^{2}\right) & =\frac{1}{(n-4)^{2}} F_{j}^{(-2)}\left(m_{1}^{2}, m_{2}^{2}, m_{3}^{2}, p^{2}\right)+\frac{1}{(n-4)} F_{j}^{(-1)}\left(m_{1}^{2}, m_{2}^{2}, m_{3}^{2}, p^{2}\right) \\
& +F_{j}^{(0)}\left(m_{1}^{2}, m_{2}^{2}, m_{3}^{2}, p^{2}\right)+\cdots, \quad j=0,1,2,3
\end{aligned}
$$

The calculation of the MI for $m_{3}=0$ presented here make use of two systems of differential equations satisfied by the MI of the sunrise diagram given in [3]. One of them in the variable $p^{2}$

$$
\begin{aligned}
p^{2} \frac{\partial}{\partial p^{2}} F_{0} & =(n-3) F_{0}+m_{1}^{2} F_{1}+m_{2}^{2} F_{2}+m_{3}^{2} F_{3} \\
p^{2} D\left(m_{1}^{2}, m_{2}^{2}, m_{3}^{2}, p^{2}\right) \frac{\partial}{\partial p^{2}} F_{i} & =\sum_{j=0}^{3} M_{i, j} F_{j}+T_{i}, i=1,2,3,
\end{aligned}
$$

where the explicit form of functions $T_{i}$ (expressed as functions of $T$ ) and $M_{i, j}$ (polynomials of $\left.p^{2}, m_{1}^{2}, m_{2}^{2}, m_{3}^{2}\right)$ can be found in [3]. The function $D$ is defined by

$$
\begin{aligned}
D\left(m_{1}^{2}, m_{2}^{2}, m_{3}^{2}, p^{2}\right)=\quad & \left(p^{2}+\left(m_{1}+m_{2}+m_{3}\right)^{2}\right)\left(p^{2}+\left(m_{1}+m_{2}-m_{3}\right)^{2}\right) \\
& \left(p^{2}+\left(m_{1}-m_{2}+m_{3}\right)^{2}\right)\left(p^{2}+\left(m_{1}-m_{2}-m_{3}\right)^{2}\right),
\end{aligned}
$$

and the aforementioned function $T$ by 


$$
T\left(n, m^{2}\right)=\int \frac{d^{n} k}{(2 \pi)^{n-2}} \frac{1}{k^{2}+m^{2}}=\frac{m^{n-2}}{(n-2)(n-4)} C(n)
$$

where

$$
C(n)=(2 \sqrt{\pi})^{(4-n)} \Gamma\left(3-\frac{n}{2}\right) \quad \text { and } \quad C(4)=1
$$

The second system of differential equations, with $m_{3}^{2}$ as a variable, can be easily obtained using expressions presented in the Appendix of [3]. They represent $A\left(n, m_{1}^{2}, m_{2}^{2}, m_{3}^{2}, p^{2},-3,-1,-1,0,0\right)$ and $A\left(n, m_{1}^{2}, m_{2}^{2}, m_{3}^{2}, p^{2},-1,-2,-2,0,0\right)$ as linear combinations of the MI. Remembering that

$$
\begin{aligned}
& A\left(n, m_{1}^{2}, m_{2}^{2}, m_{3}^{2}, p^{2},-1,-2,-2,0,0\right)=-\frac{\partial F_{2}\left(n, m_{1}^{2}, m_{2}^{2}, m_{3}^{2}, p^{2}\right)}{\partial m_{3}^{2}} \\
& A\left(n, m_{1}^{2}, m_{2}^{2}, m_{3}^{2}, p^{2},-1,-1,-3,0,0\right)=-\frac{1}{2} \frac{\partial F_{3}\left(n, m_{1}^{2}, m_{2}^{2}, m_{3}^{2}, p^{2}\right)}{\partial m_{3}^{2}}
\end{aligned}
$$

etc.

they are also differential equations in the variable $m_{3}^{2}$ (or in any other mass by means of permutation of masses). They can be written as

$$
\begin{array}{r}
\frac{\partial}{\partial m_{3}^{2}} F_{0}\left(n, m_{1}^{2}, m_{2}^{2}, m_{3}^{2}, p^{2}\right)=A_{0,0} F_{0}\left(n, m_{1}^{2}, m_{2}^{2}, m_{3}^{2}, p^{2}\right)+A_{0,1} F_{1}\left(n, m_{1}^{2}, m_{2}^{2}, m_{3}^{2}, p^{2}\right) \\
+A_{0,2} F_{2}\left(n, m_{1}^{2}, m_{2}^{2}, m_{3}^{2}, p^{2}\right)+A_{0,3} F_{3}\left(n, m_{1}^{2}, m_{2}^{2}, m_{3}^{2}, p^{2}\right)+B_{0} \\
\frac{\partial}{\partial m_{3}^{2}} F_{i}\left(n, m_{1}^{2}, m_{2}^{2}, m_{3}^{2}, p^{2}\right)=A_{i, 0} F_{0}\left(n, m_{1}^{2}, m_{2}^{2}, m_{3}^{2}, p^{2}\right)+A_{i, 1} F_{1}\left(n, m_{1}^{2}, m_{2}^{2}, m_{3}^{2}, p^{2}\right) \\
+A_{i, 2} F_{2}\left(n, m_{1}^{2}, m_{2}^{2}, m_{3}^{2}, p^{2}\right)+A_{i, 3} F_{3}\left(n, m_{1}^{2}, m_{2}^{2}, m_{3}^{2}, p^{2}\right)+B_{i}
\end{array}
$$

where $i=1,2,3$. We do not present here for short the explicit form of the coefficients $A_{i, j}$ and $B_{i}$ as they can be easily found from [3]. 
To obtain the values of the MI at $m_{3}^{2}=0$ we write the expansion of $F_{0}\left(n, m_{1}^{2}, m_{2}^{2}, m_{3}^{2}, p^{2}\right)$ at that point (the expansions of the other master integrals are obtained using Eq.(8))

$$
F_{0}\left(n, m_{1}^{2}, m_{2}^{2}, m_{3}^{2}, p^{2}\right)=\sum_{\alpha_{i} \in A}\left(m_{3}^{2}\right)^{\alpha_{i}} \cdot \sum_{k=0}^{\infty} F_{k}^{\alpha_{i}}\left(m_{3}^{2}\right)^{k}
$$

where $A$ is a set of all allowed values of $\alpha_{i}$. The allowed values of $\alpha_{i}$ one can easily get putting Eq.(10) and its $m_{i}^{2}$ derivatives into Eq.(9). We have found that there exist only two allowed values of the powers $\alpha_{i}$ and the expansion of $F_{0}\left(n, m_{1}^{2}, m_{2}^{2}, m_{3}^{2}, p^{2}\right)$ reads

$$
F_{0}\left(n, m_{1}^{2}, m_{2}^{2}, m_{3}^{2}, p^{2}\right)=\sum_{k=0}^{\infty} F_{k}^{r}\left(m_{3}^{2}\right)^{k}+\left(m_{3}^{2}\right)^{(n-2) / 2} \cdot \sum_{k=0}^{\infty} F_{k}^{s}\left(m_{3}^{2}\right)^{k}
$$

It consists of two independent series, the regular one denoted as $r$ and the singular one denoted as $s$. It means that the $m_{3}^{2}=0$ point is a regular singular point [11] of that equations. The equations Eq.(9) provide also with the following differential equations

$$
\begin{aligned}
R^{2}\left(m_{1}^{2}, m_{2}^{2},-p^{2}\right) \frac{\partial}{\partial m_{2}^{2}} F_{0}^{s} & =(n-3)\left(m_{2}^{2}-m_{1}^{2}+p^{2}\right) F_{0}^{s}-\frac{C^{2}(n)\left(m_{1}^{2}\right)^{(n-2) / 2}}{(n-2)(n-4)^{2}} \\
& +\frac{C^{2}(n)\left(m_{2}^{2}\right)^{(n-4) / 2}\left(m_{1}^{2}+m_{2}^{2}+p^{2}\right)}{2(n-2)(n-4)^{2}} \\
R^{2}\left(m_{1}^{2}, m_{2}^{2},-p^{2}\right) \frac{\partial}{\partial m_{1}^{2}} F_{0}^{s} & =(n-3)\left(m_{1}^{2}-m_{2}^{2}+p^{2}\right) F_{0}^{s}-\frac{C^{2}(n)\left(m_{2}^{2}\right)^{(n-2) / 2}}{(n-2)(n-4)^{2}} \\
& +\frac{C^{2}(n)\left(m_{1}^{2}\right)^{(n-4) / 2}\left(m_{1}^{2}+m_{2}^{2}+p^{2}\right)}{2(n-2)(n-4)^{2}},
\end{aligned}
$$

where

$$
R^{2}\left(-p^{2}, m_{1}^{2}, m_{2}^{2}\right)=p^{4}+m_{1}^{4}+m_{2}^{4}+2 m_{1}^{2} p^{2}+2 m_{2}^{2} p^{2}-2 m_{1}^{2} m_{2}^{2}
$$

On the first sight it is not necessary to know the singular part when looking for the value of the MI at $m_{3}=0$ as it vanishes in that limit. However as it will be clear from the discussion below knowledge of its poles in $(n-4)$ provides an essential simplification in the calculation of the regular part. 
The equations Eq.(12) can in principle be solved, but it is much easier to use system of equations Eq. (国) with $p^{2}$ as an independent variable for calculation of $F_{0}^{s}$. Substituting $F_{0}\left(n, m_{1}^{2}, m_{2}^{2}, m_{3}^{2}, p^{2}\right)$ and other MI with their mass expansions in the system of equations Eq.(四) we have found that $F_{0}^{s}$ satisfies the following differential equation

$$
\begin{aligned}
\frac{\partial}{\partial p^{2}} F_{0}^{s} & =\frac{1}{2 p^{2}}(n-4) F_{0}^{s}-\frac{1}{p^{2} R^{2}\left(m_{1}^{2}, m_{2}^{2},-p^{2}\right)}\left[(n-3)\left(p^{2}\left(m_{1}^{2}+m_{2}^{2}\right)+\left(m_{1}^{2}-m_{2}^{2}\right)^{2}\right) F_{0}^{s}\right. \\
& +\frac{C^{2}(n)}{2(n-2)(n-4)^{2}}\left(m_{1}^{2}\right)^{(n-2) / 2}\left(p^{2}-m_{2}^{2}+m_{1}^{2}\right) \\
& \left.+\frac{C^{2}(n)}{2(n-2)(n-4)^{2}}\left(m_{2}^{2}\right)^{(n-2) / 2}\left(p^{2}-m_{1}^{2}+m_{2}^{2}\right)\right]
\end{aligned}
$$

We have found a solution of that differential equation in a form expanded around $n=4$. Only pole parts of the function were calculated as only the poles are necessary for our purpose of calculating the values of the MI at $m_{3}=0$. The solution reads

$$
\begin{aligned}
F_{0}^{s}= & -\frac{C^{2}(n)}{4(n-4)^{2}}+\frac{C^{2}(n)}{16(n-4)}\left[6-\log \left(m_{1}^{2}\right)-\log \left(m_{2}^{2}\right)+\frac{m_{1}^{2}-m_{2}^{2}}{p^{2}} \log \left(\frac{m_{1}^{2}}{m_{2}^{2}}\right)\right] \\
& +\frac{1}{(n-4)} R\left(m_{1}^{2}, m_{2}^{2},-p^{2}\right)\left[\frac{C^{2}(n)}{8 p^{2}} \log (t)-\frac{S_{l}}{p^{2}}\right]+\mathcal{O}\left((n-4)^{0}\right)
\end{aligned}
$$

where $S_{l}$ is an integration constant. The unknown function of $m_{1}$ and $m_{2}$, which remains after $p^{2}$ integration, was reduced to a single numerical constant $S_{l}$ using the equations Eq.(12).

The system of equations Eq.(9) gives no information about $F_{0}^{r}$, provides however an expression for the second coefficient in the regular series. It can be expressed as a linear combination of $F_{0}^{r}$ and its derivatives

$$
\begin{aligned}
F_{1}^{r}= & \frac{1}{R^{2}\left(m_{1}^{2}, m_{2}^{2},-p^{2}\right)}\left(\frac{3}{(n-4)}\left(n-\frac{8}{3}\right)(n-3)\left(m_{2}^{2}+m_{1}^{2}+p^{2}\right) F_{0}^{r}\right. \\
& -\frac{4}{(n-4)} m_{1}^{2}(n-3)\left(p^{2}+m_{1}^{2}\right) \frac{\partial}{\partial m_{1}^{2}} F_{0}^{r}-\frac{4}{(n-4)} m_{2}^{2}(n-3)\left(p^{2}+m_{2}^{2}\right) \frac{\partial}{\partial m_{2}^{2}} F_{0}^{r} \\
& \left.+\frac{1}{(n-4)^{3}}\left(m_{1}^{2}\right)^{(n-2) / 2}\left(m_{2}^{2}\right)^{(n-2) / 2} C^{2}(n)\right) .
\end{aligned}
$$


Putting the expansion Eq.(11) into the equations Eq.(4) one finds a system of three differential equations satisfied by $F_{0}^{r}, \frac{\partial}{\partial m_{1}^{2}} F_{0}^{r}$ and $\frac{\partial}{\partial m_{2}^{2}} F_{0}^{r}$

$$
\begin{aligned}
& \frac{\partial}{\partial p^{2}} F_{0}^{r}=\frac{1}{p^{2}}(n-4) F_{0}^{r}+\frac{1}{p^{2}} F_{0}^{r}-\frac{m_{1}^{2}}{p^{2}} \frac{\partial}{\partial m_{1}^{2}} F_{0}^{r}-\frac{m_{2}^{2}}{p^{2}} \frac{\partial}{\partial m_{2}^{2}} F_{0}^{r} \\
& \frac{\partial}{\partial p^{2}} \frac{\partial}{\partial m_{1}^{2}} F_{0}^{r}=\frac{1}{R^{2}\left(m_{1}^{2}, m_{2}^{2},-p^{2}\right)}\left[\frac{p^{2}+m_{1}^{2}-m_{2}^{2}}{2 p^{2}}\left(4+7(n-4)+3(n-4)^{2}\right) F_{0}^{r}\right. \\
& +(n-4) \frac{\partial}{\partial m_{1}^{2}} F_{0}^{r}\left(\frac{2 m_{1}^{2} m_{2}^{2}}{p^{2}}-\frac{3 m_{1}^{4}}{2 p^{2}}-\frac{m_{2}^{4}}{2 p^{2}}+\frac{p^{2}}{2}-m_{1}^{2}\right) \\
& +\frac{\partial}{\partial m_{1}^{2}} F_{0}^{r}\left(\frac{3 m_{1}^{2} m_{2}^{2}}{p^{2}}-\frac{2 m_{1}^{4}}{p^{2}}-\frac{m_{2}^{4}}{p^{2}}-2 m_{1}^{2}-m_{2}^{2}\right) \\
& +(n-3) \frac{\partial}{\partial m_{2}^{2}} F_{0}^{r}\left(-\frac{m_{1}^{2} m_{2}^{2}}{p^{2}}+\frac{m_{2}^{4}}{p^{2}}-3 m_{2}^{2}\right) \\
& \left.+\frac{C^{2}(n)\left(m_{1}^{2}\right)^{(n-4) / 2}\left(m_{2}^{2}\right)^{(n-2) / 2}\left(-p^{2}+m_{1}^{2}-m_{2}^{2}\right)}{4(n-4)^{2} p^{2}}\right] \\
& \frac{\partial}{\partial p^{2}} \frac{\partial}{\partial m_{2}^{2}} F_{0}^{r}=\frac{1}{R^{2}\left(m_{1}^{2}, m_{2}^{2},-p^{2}\right)}\left[\frac{p^{2}-m_{1}^{2}+m_{2}^{2}}{2 p^{2}}\left(4+7(n-4)+3(n-4)^{2}\right) F_{0}^{r}\right. \\
& +(n-4) \frac{\partial}{\partial m_{2}^{2}} F_{0}^{r}\left(\frac{2 m_{1}^{2} m_{2}^{2}}{p^{2}}-\frac{m_{1}^{4}}{2 p^{2}}-\frac{3 m_{2}^{4}}{2 p^{2}}+\frac{p^{2}}{2}-m_{2}^{2}\right) \\
& +\frac{\partial}{\partial m_{2}^{2}} F_{0}^{r}\left(\frac{3 m_{1}^{2} m_{2}^{2}}{2 p^{2}}-\frac{m_{1}^{4}}{p^{2}}-\frac{2 m_{2}^{4}}{p^{2}}-m_{1}^{2}-2 m_{2}^{2}\right) \\
& +(n-3) \frac{\partial}{\partial m_{1}^{2}} F_{0}^{r}\left(-\frac{m_{1}^{2} m_{2}^{2}}{p^{2}}+\frac{m_{1}^{4}}{p^{2}}-3 m_{1}^{2}\right) \\
& \left.+\frac{C^{2}(n)\left(m_{1}^{2}\right)^{(n-2) / 2}\left(m_{2}^{2}\right)^{(n-4) / 2}\left(-p^{2}-m_{1}^{2}+m_{2}^{2}\right)}{4(n-4)^{2} p^{2}}\right] \text {, }
\end{aligned}
$$

Trying to find $F_{0}^{r}$ from Eq.(17) only would mean to solve a third order differential equation satisfied by $F_{0}^{r}$. That task can be simplified enormously by careful examination of the Eq.(16). One finds from it that in the $(n-4)$ expansion of $F_{1}^{r}$ the terms $\sim(n-4)^{0}$ of $F_{0}^{r}$ contribute to pole terms of $F_{1}^{r}$. As a result, knowing the pole terms of the singular part of the expansion (Eq.(15)) and the exact pole terms of the complete master integral $F_{0}$ [3], we can find an additional relation between $F_{0}^{r}$ and its mass derivatives. Defining the $(n-4)$ expansion of the $F_{0}^{r}$ by 


$$
F_{0}^{r}=\frac{1}{(n-4)^{2}} F_{0,-2}^{r}+\frac{1}{(n-4)} F_{0,-1}^{r}+F_{0,0}^{r}+\mathcal{O}(n-4)
$$

one finds

$$
\begin{array}{r}
0=\left(m_{1}^{2}+m_{2}^{2}+p^{2}\right) F_{0,0}^{r}-m_{1}^{2}\left(p^{2}+m_{1}^{2}\right) \frac{\partial}{\partial m_{1}^{2}} F_{0,0}^{r}-m_{2}^{2}\left(p^{2}+m_{2}^{2}\right) \frac{\partial}{\partial m_{2}^{2}} F_{0,0}^{r} \\
+R\left(m_{1}^{2}, m_{2}^{2},-p^{2}\right)\left(\frac{C^{2}(n)}{32} \log (t)-\frac{S_{l}}{4}\right)\left(p^{2}+2 m_{1}^{2}+2 m_{2}^{2}+\frac{1}{p^{2}}\left(m_{1}^{2}-m_{2}^{2}\right)^{2}\right) \\
+C^{2}(n)\left(\frac{\left(p^{2}\right)^{2}}{128}\left(13-2 \log \left(m_{1}^{2}\right)-2 \log \left(m_{2}^{2}\right)\right)+\frac{p^{2}}{128}\left(41\left(m_{1}^{2}+m_{2}^{2}\right)\right.\right. \\
\left.-\log \left(m_{1}^{2}\right)\left(14 m_{1}^{2}+6 m_{2}^{2}\right)-\log \left(m_{2}^{2}\right)\left(6 m_{1}^{2}+14 m_{2}^{2}\right)\right)+\frac{1}{64 p^{2}}\left(m_{2}^{4}\left(3 m_{1}^{2}-m_{2}^{2}\right)\right. \\
\left.-m_{1}^{4}\left(3 m_{2}^{2}-m_{1}^{2}\right)\right)\left(\log \left(m_{1}^{2}\right)-\log \left(m_{2}^{2}\right)\right)+\frac{m_{1}^{2} m_{2}^{2}}{32}\left(\log \left(m_{1}^{2}\right)+\log \left(m_{2}^{2}\right)\right)^{2} \\
+\frac{1}{32}\left(12 m_{1}^{2} m_{2}^{2}+7 m_{1}^{4}+7 m_{2}^{4}\right)-\frac{\log \left(m_{1}^{2}\right)}{64}\left(12 m_{1}^{2} m_{2}^{2}+5 m_{1}^{4}+3 m_{2}^{4}\right) \\
\left.-\frac{\log \left(m_{2}^{2}\right)}{64}\left(12 m_{1}^{2} m_{2}^{2}+3 m_{1}^{4}+5 m_{2}^{4}\right)\right)
\end{array}
$$

where

$$
t=\frac{\sqrt{p^{2}+\left(m_{1}+m_{2}\right)^{2}}-\sqrt{p^{2}+\left(m_{1}-m_{2}\right)^{2}}}{\sqrt{p^{2}+\left(m_{1}+m_{2}\right)^{2}}+\sqrt{p^{2}+\left(m_{1}-m_{2}\right)^{2}}} .
$$

It allows us to reduce the third order equation obtained from Eq.(17) to a relatively simple second order differential equation

$$
\begin{aligned}
\frac{\partial^{2}}{\partial\left(p^{2}\right)^{2}} F_{0,0}^{r}= & -\frac{2}{p^{2}} \frac{\partial}{\partial p^{2}} F_{0,0}^{r}-\frac{1}{\left(p^{2}\right)^{2}} R\left(m_{1}^{2}, m_{2}^{2},-p^{2}\right)\left(\frac{C^{2}(n)}{16} \log (t)-\frac{1}{2} S_{l}\right) \\
& -\frac{C^{2}(n)}{32\left(p^{2}\right)^{2}}\left[\left(m_{1}^{2}-m_{2}^{2}\right) \log \left(\frac{m_{1}^{2}}{m_{2}^{2}}\right)-\log \left(m_{2}^{2}\right) p^{2}-\log \left(m_{1}^{2}\right) p^{2}+\frac{7 p^{2}}{2}\right]
\end{aligned}
$$

Its integration is elementary giving dilogarithms as the most 'complicated' functions and the solution after all integration constants were fixed reads 


$$
\begin{aligned}
& F_{0}^{(0)}\left(m_{1}^{2}, m_{2}^{2}, 0, p^{2}\right)=F_{0,0}^{r}= \\
& C^{2}(n)\left[\frac{m_{1}^{2} m_{2}^{2} \log ^{2}(t)}{16 p^{2}}+\frac{1}{64 p^{2}}\left(-m_{1}^{2} m_{2}^{2} \log ^{2}\left(t_{1}^{2}\right)+\left(m_{1}^{4}-m_{2}^{4}\right) \log \left(t_{1}^{2}\right)\right)\right. \\
+ & \frac{p^{2}}{64}\left(\log \left(m_{2}^{2}\right)+\log \left(m_{1}^{2}\right)-\frac{13}{2}\right)-\frac{\log (t)}{32\left(t-t_{1}\right)}\left(\frac{m_{1}^{3}}{m_{2}}+m_{1} m_{2}\right) \\
- & \frac{\log (t)}{32\left(t-t_{2}\right)}\left(\frac{m_{2}^{3}}{m_{1}}+m_{1} m_{2}\right)-\frac{\log (t)}{32}\left(R\left(m_{1}^{2}, m_{2}^{2},-p^{2}\right)+2 m_{1}^{2}+2 m_{2}^{2}\right) \\
+ & \frac{\log ^{2}(t)-1}{32}\left(m_{1}^{2}+m_{2}^{2}\right)+\frac{m_{2}^{2}-m_{1}^{2}}{16}\left(\operatorname{Li}_{2}\left(1-\frac{t}{t_{1}}\right)-\operatorname{Li}_{2}\left(1-\frac{t}{t_{2}}\right)\right) \\
+ & \frac{2 \log \left(t_{1}\right)\left(m_{1}^{2}-m_{2}^{2}\right)+m_{1}^{2}+m_{2}^{2}}{32} \log \left(\frac{\left(t_{1}-t\right)\left(t_{2}-t\right)}{p^{2}}\right) \\
+ & \frac{\log ^{2}\left(m_{1}^{2}\right)}{64}\left(13 m_{1}^{2}+m_{2}^{2}\right)+\frac{\log \left(m_{2}^{2}\right)}{64}\left(13 m_{2}^{2}+m_{1}^{2}\right)-\frac{\log ^{2}\left(m_{1}^{2}\right)}{128}\left(5 m_{1}^{2}+m_{2}^{2}\right) \\
- & \left.\frac{\log ^{2}\left(m_{2}^{2}\right)}{128}\left(5 m_{2}^{2}+m_{1}^{2}\right)-\left(\frac{\log \left(m_{1}^{2}\right) \log \left(m_{2}^{2}\right)}{64}+\frac{5}{32}\right)\left(m_{1}^{2}+m_{2}^{2}\right)\right],
\end{aligned}
$$

where

$$
t_{1}=\frac{m_{1}}{m_{2}} \quad \text { and } \quad t_{2}=\frac{m_{2}}{m_{1}}
$$

The constant $S_{l}$ was fixed using the analytically known [3] result for $F_{0}\left(n, 0,0, m_{3}^{2}, p^{2}\right)$, while two unknown functions of $m_{1}$ and $m_{2}$ coming from the integration of the second order equation Eq.(21) where fixed from known [3] results for $F_{0}\left(n, m_{1}^{2}, m_{2}^{2}, m_{3}^{2}, 0\right)$ and $\frac{\partial}{\partial p^{2}} F_{0}\left(n, m_{1}^{2}, m_{2}^{2}, m_{3}^{2}, p^{2}\right)\left(p^{2}=0\right)$. The value of $p^{2}$ is to be understood, whenever necessary, as $p^{2}-i \epsilon$, with $\epsilon$ an infinitesimally small positive constant.

Having $F_{0}^{(0)}\left(m_{1}^{2}, m_{2}^{2}, 0, p^{2}\right)$ one can easily get other two independent MI: $F_{1}^{(0)}\left(m_{1}^{2}, m_{2}^{2}, 0, p^{2}\right)$ and $F_{2}^{(0)}\left(m_{1}^{2}, m_{2}^{2}, 0, p^{2}\right)$. We present here only one of them as the other one can be found by an exchange of the masses $m_{1}$ and $m_{2}$. It reads

$$
\begin{aligned}
& F_{1}^{(0)}\left(m_{1}^{2}, m_{2}^{2}, 0, p^{2}\right)=-\frac{\partial}{\partial m_{1}^{2}} F_{0,0}^{r}= \\
& \quad-C^{2}(n)\left[\frac{1}{32}+\frac{R\left(m_{1}^{2}, m_{2}^{2},-p^{2}\right) \log (t)}{16 p^{2}}+\frac{\log \left(t_{1}^{2}\right)}{32 p^{2}}\left(m_{1}^{2}-\frac{m_{2}^{2}}{2}\left(\log \left(t_{1}^{2}\right)+1\right)\right)\right.
\end{aligned}
$$




$$
\begin{aligned}
& +\frac{\log (t)}{32}\left(t_{2}^{2}-2+\frac{\log (t)}{p^{2}}\left(p^{2}+2 m_{2}^{2}\right)\right)-\frac{t_{2}^{2}}{64}\left(\log \left(m_{1}^{2}\right)+\log \left(m_{2}^{2}\right)\right) \\
& -\frac{\log \left(m_{2}^{2}\right)}{128}\left(2 \log \left(m_{1}^{2}\right)+\log \left(m_{2}^{2}\right)\right)+\log \left(m_{1}^{2}\right)\left(\frac{1}{8}-\frac{5 \log \left(m_{1}^{2}\right)}{128}\right) \\
& \left.+\frac{1}{32}\left(2-t_{2}^{2}+\log \left(t_{1}^{2}\right)\right) \log \left(\frac{\left(t_{1}-t\right)\left(t_{2}-t\right)}{p^{2}}\right)+\frac{1}{16}\left(\operatorname{Li}_{2}\left(1-\frac{t}{t_{2}}\right)-\operatorname{Li}_{2}\left(1-\frac{t}{t_{1}}\right)\right)\right] .
\end{aligned}
$$

We have tested numerically the results of Eq. 221) and Eq.(23) against results obtained by newly developed program [12], which solves numerically the system of differential equations Eq.(化). In the program one cannot put however one of the masses to zero, though very small values of the masses are allowed. Putting sufficiently small masses to eliminate its influence on the result an agreement of 10 decimal digits was found between the numerical program and the analytical results (Eq.(22) and Eq.(23)).

\section{Conclusions.}

An extensive use of the differential equation method allowed to find closed analytical expressions for the sunrise master integrals with one mass equal to zero, two arbitrary masses and an arbitrary external four momentum.

\section{Acknowledgments.}

One of us (HC) is grateful to M. Caffo and E. Remiddi for valuable discussions concerning the sunrise graph.

\section{References}

[1] F.V. Tkachov, Phys. Lett.B 100 (1981) 65; K.G. Chetyrkin and F.V. Tkachov, Nucl. Phys.B 192 (1981) 159.

[2] F.A. Berends, M. Böhm, M. Buza and R. Scharf, Z. Phys.C 63 (1994) 227.

[3] M. Caffo, H. Czyż, S. Laporta and E. Remiddi, Nuovo Cim.A 111 (1998) 365, hepth/9805118.

[4] F.A. Berends, A.I.Davydychev, N.I. Ussyukina, Phys. Lett.B 426 (1998) 95, hepph/9712209. 
[5] M. Caffo, H. Czyż and E. Remiddi, Nucl. Phys.B 581 (2000) 274, hep-ph/9912501.

[6] A.I. Davydychev and V.A. Smirnov, Nucl. Phys.B 554 (1999) 391, hep-ph/9903328.

[7] S. Groote and A.A. Pivovarov, Nucl. Phys. B 580 (2000) 459, hep-ph/0003115.

[8] M. Caffo, H. Czyż and E. Remiddi, Nucl. Phys.B 611 (2001) 503, hep-ph/0103014.

[9] J. Fleisher, M.Yu. Kalmykov and A.V. Kotikov, Phys. Lett.B 462 (1999) 169, hep$\mathrm{ph} / 9905249$.

[10] M. Argeri, P. Mastrolia and E. Remiddi, hep-ph/0202123.

[11] E.L. Ince, Ordinary differential equations, Dover Publications, New York, 1956.

[12] M. Caffo, H. Czyż and E. Remiddi, hep-ph/0203256. 